\title{
Nivel de mercurio en cabello de niños peruanos expuestos en una zona minera de Arequipa y de la ciudad de Lima
}

\section{Level of mercury in hair of peruvian children exposed in a mining area of Arequipa and Lima city}

\author{
Villanueva-Ponce $A^{*}$, Adserias-Garriga $\mathrm{MJ}^{* *}$, Chimenos-Küstner E***
}

\section{RESUMEN}

Objetivo: El propósito del estudio fue comparar los niveles de mercurio en cabello en dos muestras de niños peruanos provenientes de dos zonas geográficas distintas: una zona rural minera y una zona urbana.

Material y métodos: La zona minera correspondió al poblado de Mollehuaca (Arequipa), de donde se obtuvieron 52 muestras de cabello; la zona urbana no expuesta a minería correspondió al distrito de Los Olivos (Lima), de donde se obtuvieron 40 muestras. Se determinó el nivel de mercurio con la técnica estandarizada de plasma de inducción acoplado con un espectrómetro de masas (ICP-MS).

Resultados: En la zona rural, en promedio, se obtuvo un nivel de mercurio de 7,47 ppb $\pm 26,9$. En la zona urbana, el nivel de mercurio alcanzó un promedio de $2,47 \mathrm{ppb} \pm 1,28$. No se encontraron diferencias significativas en el nivel de mercurio según edad o género.

Conclusión: Se concluye que el grupo expuesto a minería aurífera informal y de zona rural presentó niveles de mercurio en cabello en promedio mayores a los del grupo de zona urbana, pero esta diferencia no fue significativa $(p=0,188, \alpha=0,05)$.

Palabras clave: Cabello, mercurio, análisis toxicológico, minería.

\section{SUMMARY}

Objective: The aim of this study is to compare the mercury levels tested in two groups of children from different locations from Peru: a mining countryside and a metropolitan area.

Methods: The rural area was Mollehuaca (Arequipa), where children are exposed to mercury by artisanal mining. Fifty-two hair samples from children from the rural area were obtained. The urban area was District of Olives (Lima), where there is not mercury exposure by mining. Forty hair samples from children from the urban area were obtained. Mercury level was determined by induction coupled plasma mass spectrometer (ICP-MS) standard technique.

Results: In the rural area, an average of level of mercury $7.47 \mathrm{ppb} \pm 26.9$ was obtained; in urban areas the mercury level reached an average of $2.47 \mathrm{ppb} \pm 1.28$. Not significant differences in the level of mercury according to age or gender were found.

* Posgrado de Medicina Bucal.

** Ex Profesora Asociada de Medicina Bucal.

*** Profesor Titular de Medicina Bucal.

Facultad de Odontología. Universidad de Barcelona. España. 
Conclusion: It is concluded that the group exposed to rural and informal gold mining presented an average of mercury levels in hair higher than those of the urban group, but this difference was not significant $(\mathrm{p}=0.188$, $\alpha=0.05)$.

Key words: Hair, mercury, toxicological analysis, mining.

Fecha de recepción: 13 de mayo de 2014.

Aceptado para publicación: 20 de junio de 2014.

Villanueva-Ponce A, Adserias-Garriga MJ, Chimenos-Küstner E. Nivel de mercurio en cabello de niños peruanos expuestos en una zona minera de Arequipa y de la ciudad de Lima. Av. Odontoestomatol 2015; 31 (2): $85-$ 90.

\section{INTRODUCCIÓN}

En el Perú, durante las últimas décadas se ha observado un creciente desarrollo de la actividad minera, especialmente la aurífera, que no cumple con la normativa de control de desechos, en especial, de mercurio, elemento perjudicial para la salud de la población. Al ser inhalado afecta principalmente al sistema nervioso, causando atrofia de la corteza cerebral sensorial, deterioro audiovisual y parálisis cerebral. La presencia de mercurio en el organismo puede causar trastornos de aprendizaje y conducta. Generalmente los niños tienen más riesgo de intoxicarse que los adultos (1).

El presente estudio se realizó en la comunidad minera de Mollehuaca, perteneciente al distrito de Huanuhuanu, provincia de Caravelí del departamento de Arequipa, ubicado en la sierra sur del país. Está a una altura de 1.200 metros sobre el nivel del mar. Mollehuaca se ubica entre quebradas áridas y cerros, con yacimientos mineros de oro y cobre, que se extiende en los departamentos de Ica y Arequipa, con una producción aproximada de oro de 7,5 TM/ año para los lavaderos (2).

La minería aurífera artesanal se ha convertido en un medio de subsistencia para familias de la zona e involucra a cerca de 1.460 personas que reciben influencia directa e indirecta de esta actividad (2). Como este tipo de minería se desarrolla con técnicas rudimentarias, empleando mercurio metálico en los procesos, y en virtud de que diariamente los ver- tidos de la minería son arrojados al suelo sin ningún tipo de tratamiento, existe un alto riesgo de contaminación por mercurio, que no debería existir en zonas sin este factor de riesgo como Lima.

A tenor de la formación del grupo investigador (ámbito odontoestomatológico), en un principio se pretendía determinar la concentración de mercurio en saliva. De hecho, se hizo una primera recolección de muestras de saliva, que luego hubo que desechar por problemas de conservación, almacenamiento, transporte y dificultad de manipulación y posterior estudio en España. Por esa razón y amparados en un mayor soporte científico hallado en la literatura, finalmente se optó por estudiar la concentración en cabello (mucho más fácil de manipular y sin los problemas planteados por el fluido salival).

Bajo estas premisas, el objetivo del estudio consistió en comparar los niveles de mercurio en cabello en dos muestras de niños peruanos provenientes de dos zonas geográficas distintas: una zona rural minera y una zona urbana.

\section{MATERIAL Y MÉTODOS}

El análisis de mercurio se realizó en cabello, porque representa una muestra simple, integradora y no invasiva con la que estimar la exposición promedio a largo plazo (3). Por otra parte, el cabello constituye una herramienta útil para monitorizar el mercurio en exposiciones de tipo ocupacional (4). 


\section{Población estudiada}

Fueron niños habitantes en zonas rurales mineras de la región de Arequipa y en la zona urbana no expuesta a minería de la región de Lima. La muestra de cabellos analizados correspondió a 92 niños de ambos grupos, de los cuales 52 habitaban en el poblado de Mollehuaca, distrito de Huanuhuanu, Caravelí, al sur de Arequipa, y 40 niños residentes en la ciudad de Lima, distrito de los Olivos, como muestra control. Se incluyó a 46 niñas y 46 niños, de edades comprendidas entre los 6 y 12 años. El muestreo fue de tipo no probabilístico, intencional, de sujetos voluntarios, pues los niños expuestos fueron tomados en cuenta según su disponibilidad y autorización de los padres.

\section{Recolección de las muestras de cabello}

Se recolectaron las muestras de cabello entre los meses de febrero y mayo del 2011. Para ello, se recortaron con una tijera aproximadamente $100 \mathrm{mg}$ de cabello distal de la región occipital, al ras del cuero cabelludo, a cada niño de cada uno de los grupos. Cada muestra se depositó en un envase de plástico rotulado, donde se conservó hasta el momento del análisis.

Conjuntamente con la recolección de la muestra de cabello de cada niño, se registraron los datos relativos a sexo y edad. Todas las muestras fueron tomadas bajo consentimiento y conocimiento de los padres o tutores de los niños involucrados.

\section{Tratamiento de la muestra y análisis}

Una vez en el laboratorio, las muestras de cabello fueron lavadas con detergente (Tritón X-100) y agua desionizada. El mercurio se analizó por la técnica de plasma de inducción acoplada con un espectrómetro de masas, empleando el equipo Perkin Elmer ELAN 6000, previa digestión con ácido nítrico. Se determinó la relación peso/volumen.

\section{Análisis de datos}

Para el análisis estadístico de los niveles de mercurio en las muestras de cabello de zona rural y zona urba- na se emplearon medidas de tendencia central (media y mediana) y como medida de dispersión la desviación estándar, para efectos de comparación de los niveles de mercurio en ambos grupos, inicialmente de forma global y luego por edades y género.

\section{RESULTADOS Y DISCUSIÓN}

Los niveles de mercurio en muestras de cabello, en la población de Mollehuaca llegan a valores promedio de 7,47 ppb y en la ciudad de Lima estos valores son en promedio $2,47 \mathrm{ppb}$, inferiores a los de zona rural. Sin embargo, la mitad de los niños de la zona rural tienen niveles inferiores de 2,15 ppb. La dispersión en los resultados de los niños de la zona rural es elevada (26,91 ppb), lo que indica que existen algunos niños con niveles muy altos de mercurio en cabello, por lo que el error típico de la media llega a $3,73 \mathrm{ppb}$. No se encuentra diferencia significativa entre ambas poblaciones a través del "test $t$ de Student" a un nivel de confianza de $95 \%$ ( $t=-1,336 ; p=0,188$; $\alpha=0,05$ ) (tabla 1). El cabello contiene abundantes grupos sulfhidrilo y la concentración de mercurio en él es alrededor de 300 veces más que en la sangre, donde el límite máximo de concentración normal es de 3 a $4 \mu \mathrm{g} / \mathrm{dl}$ (5); se considera normal una concentración de 7 ppm de mercurio en cabello, es decir $7.000 \mathrm{ppb}$.

Esto implica que, a pesar de que los niños de la población de Mollehuaca en promedio tienen valores más elevados que los niños de Lima, estos valores no indican intoxicación por mercurio.

Al comparar por edades, las diferencias más evidentes son a los 6, 9 y 12 años. A los 6 años de edad en promedio los niveles de mercurio son $23,67 \pm 64,25$

\begin{tabular}{|l|c|c|c|c|c|c|}
\hline \multicolumn{7}{|c|}{ TABLA 1.- NIVELES DE MERCURIO EN } \\
CABELLO EN NIÑOS DE ZONA RURAL \\
EXPUESTA Y ZONA URBANA \\
\hline Grupo & $\mathbf{n}$ & $\overline{\mathrm{X}}$ & $\mathbf{M e}$ & $\mathbf{s}$ & $\boldsymbol{t}$ & Sig. Bil. \\
\hline Zona rural & 52 & 7,47 & 2,42 & 26,91 & & \\
\hline Zona urbana & 40 & 2,47 & 2,15 & 1,28 & & 0,188 \\
\hline
\end{tabular}


ppb en la zona rural y, en zona urbana, estos niveles son en promedio 2,34 $\pm 1,21 \mathrm{ppb}$. Estas diferencias no son significativas $(t=-0,995, p=0,84, \alpha=0,05)$. Hay que destacar que la elevada desviación estándar en zona rural se debe a un valor extremo en el nivel de mercurio, de 194,99 ppb. En este sentido, se comparan las medianas del nivel de mercurio a los 6 años, siendo 2,26 ppb en zona rural y 2,67 ppb en zona urbana, lo que corrobora que las diferencias no son significativas. A los 9 años, en la zona rural el promedio de mercurio en cabello es de 3,83 $\pm 4,69$ ppb, con una mediana de 2,32 ppb. En la zona urbana estos niveles son en promedio $2,70 \pm 1,50 \mathrm{ppb}$, con una mediana de 2,15 ppb. Las diferencias no son significativas $(t=-0,836 ; p=0,42 ; \alpha=0,05)$. A los 12 años, el nivel promedio de mercurio en cabello es $1,30 \pm 0,26 \mathrm{ppb}$ en la zona rural y en zona urbana es 2,11 $0,74 \mathrm{ppb}$; las diferencias tampoco son significativas en este caso $(t=-2,287 ; p=0,07 ; \alpha=$ $0,05)$. En definitiva, los hallazgos demuestran que en la zona rural los niveles de mercurio son más elevados que en la zona urbana al comparar por edades, pero esta diferencia no es significativa (Tabla 2).

Al comparar por géneros, las niñas de la zona rural presentan en promedio un nivel de mercurio de11,61 $\pm 37,78 \mathrm{ppb}$, mientras que en la zona urbana el nivel promedio es de 2,54 $\pm 1,13 \mathrm{ppb}$, no siendo estas diferencias significativas $(t=-1,223, p=$ $0,32, \alpha=0,05$. La elevada desviación estándar en niñas de zona rural también se debe al valor extremo en el nivel de mercurio de 194,99 ppb. Al comparar las medianas, el nivel de mercurio en la mitad de niñas es de 2,74 ppb en zona rural y de 2,54 ppb en zona urbana, lo que corrobora que las diferencias no son significativas. Con respecto a los niños en la zona rural el nivel promedio de mercurio es 3,32 $\pm 3,72 \mathrm{ppb}$, con una mediana de 2,22 ppb, y en la zona urbana el promedio es 2,40 $\pm 1,45 \mathrm{ppb}$, con una mediana de $2 \mathrm{ppb}$, con diferencias no significativas $(\mathrm{t}=-1,147, \mathrm{p}=0,26, \alpha=0,05)$ (Tabla 3).

\section{TABLA 2.- NIVELES DE MERCURIO EN CABELLO EN NIÑOS DE 6, 9 Y 12 AÑOS DE ZONA RURAL EXPUESTA Y ZONA URBANA}

\begin{tabular}{|c|c|c|c|c|c|c|c|}
\hline Edad & Grupo & $\mathrm{n}$ & $\mathrm{Me}$ & $\overline{\mathbf{X}}$ & $\mathbf{s}$ & $t$ & Sig. Bil. \\
\hline \multirow{2}{*}{6} & Zona rural & 9 & 2,26 & 23,67 & 64,25 & \multirow{2}{*}{$-0,995$} & \multirow{2}{*}{0,84} \\
\hline & Zona urbana & 9 & 2,67 & 2,34 & 1,21 & & \\
\hline \multirow{2}{*}{9} & Zona rural & 13 & 2,32 & 3,83 & 4,69 & \multirow{2}{*}{$-0,836$} & \multirow{2}{*}{0,42} \\
\hline & Zona urbana & 18 & 2,15 & 2,70 & 1,50 & & \\
\hline \multirow{2}{*}{12} & Zona rural & 5 & 1,27 & 1,30 & 0,26 & \multirow{2}{*}{$-2,287$} & \multirow{2}{*}{0,07} \\
\hline & Zona urbana & 4 & 2,37 & 2,11 & 0,74 & & \\
\hline
\end{tabular}

\section{TABLA 3.- NIVELES DE MERCURIO EN CABELLO SEGÚN GÉNERO EN NIÑOS DE ZONA RURAL EXPUESTA Y ZONA URBANA}

\begin{tabular}{|c|c|c|c|c|c|c|c|}
\hline Género & Grupo & $\mathrm{n}$ & $\mathrm{Me}$ & $\overline{\mathrm{X}}$ & s & $t$ & Sig. Bil. \\
\hline \multirow{2}{*}{ Femenino } & Zona rural & 26 & 2,74 & 11,61 & 37,78 & \multirow{2}{*}{$-1,223$} & \multirow{2}{*}{0,32} \\
\hline & Zona urbana & 20 & 2,54 & 2,54 & 1,13 & & \\
\hline \multirow{2}{*}{ Masculino } & Zona rural & 26 & 2,22 & 3,32 & 3,72 & \multirow{2}{*}{$-1,147$} & \multirow{2}{*}{0,26} \\
\hline & Zona urbana & 20 & 2,00 & 2,40 & 1,45 & & \\
\hline
\end{tabular}


Los valores hallados en este estudio no difieren en mucho de los valores obtenidos por Gómez y cols. (6), quienes analizaron muestras de cabellos y uñas de 90 niños escolares, entre 4 y 13 años, así como los resultados de Álvarez y cols. (7), quienes determinaron la presencia de mercurio total (cabello y orina), en pobladores de los asentamientos indígenas de "El Casabe" y "El Plomo". En ambos casos se empleó la espectrofotometría de absorción atómica al vapor frío para identificar el nivel de mercurio. Los resultados demostraron que los niveles de mercurio en cabellos no presentaron diferencias significativas en cuanto a la edad de los niños. Del mismo modo, al comparar por sexo, tampoco observaron diferencias significativas de nivel de $\mathrm{Hg}$ en cabellos de los niños y niñas estudiados. Santos y cols. (8), al evaluar los niveles de mercurio y plomo en el cabello de niños en Cubatão (Brasil), también emplearon la técnica de espectrofotometría de absorción atómica, en el estudio del cabello de 251 niños entre 1 y 10 años de edad. Obtuvieron concentraciones superiores de mercurio que los observados en el presente estudio. La concentración promedio de mercurio en el cabello fue de $0,82 \pm 0,48 \mu \mathrm{g}$, equivalente a $99,95 \pm 58,51 \mathrm{ppb}$. No hubo diferencia estadística significativa $(p \geq 0,05)$ entre los niveles medios de mercurio en el cabello, de acuerdo con la edad y el lugar de residencia. Estos resultados muestran niveles inferiores a los permitidos, al igual que en la población de Mollehuaca, a pesar de que estas zonas están expuestas a contaminación por mercurio.

En definitiva, en nuestro estudio se observa que el grupo expuesto a minería informal y de zona rural presentó niveles de concentración de mercurio en promedio mayores a los del grupo de zona urbana, pero esta diferencia no fue estadísticamente significativa. Esto cabe atribuirlo a que ambos grupos de alguna forma están expuestos a contaminación por mercurio. Los niños de Mollehuaca están expuestos por la minería informal y vacunas (9), mientras que los niños de Lima están expuestos por vivir en una zona industrializada y sobre todo por el consumo de productos marinos. Los resultados de la valoración de mercurio en cabello no demuestran exactamente la concentración de mercurio en el organismo, sino que son un referente sobre la capacidad de desintoxicación del cuerpo, una vez expuesto a este contaminante. No obstante, aun cuando los niveles de mercurio hallados en cabello no superan los límites legales admisibles para considerar una intoxicación por mercurio, las diferencias observadas constituyen un signo de alerta, que, en nuestra opinión y la de otros autores, justificaría la conveniencia de ampliar los estudios sobre este tema, con muestras poblacionales más amplias y dispersas.

\section{AGRADECIMIENTOS}

A los niños de Arequipa y Lima, por su participación. A las autoridades de los colegios, por su apoyo para el desarrollo del estudio. A la licenciada Magnolli Loza Chilquillo, por su destacada colaboración en la parte estadística.

\section{BIBLIOGRAFÍA}

1. Martí $M$, Desoille H, Scherrer J, et al. Medicina del trabajo. Factores nocivos. Barcelona: Ed. Masson 2001;292.

2. IPEC (Programa internacional para la erradicación del trabajo infantil). Evaluación intermedia. Erradicación del trabajo infantil en la comunidad minera artesanal de Mollehuaca, segunda fase. Lima: Ed. SIRTI-OIT 2001;15.

3. WHO, UNEP. Informe relativo a la información sobre los sistemas armonizados para medir la carga corporal de mercurio. WHO 2010. Disponible en URL: http://www.unep.org/hazardoussubstances/ Portals/9/Mercury/Documents/INC2/INC2_6_s. pdf.

4. Hac E, Krechniak J. Mercury concentrations in hair exposed in vitro to mercury vapor. Biol Trace Elem 1993;39(2/3):109.

5. Sepúlveda L, Agudelo L, Arengas A. El mercurio, sus implicaciones en la salud y en el ambiente. Disponible en: http://lunazul.ucaldas.edu.co/index. php?option $=$ com_contentEtask $=$ viewEid $=237 \mathcal{E}$ Itemid $=237$.

6. Gómez Rojas G. Determinación de la concentración mercurio en cabellos y uñas de niños en 
edad escolar pertenecientes a la escuela Elsa Josefina Lunar de la localidad de Guayacán, estado Sucre 2012. Ed. Universidad del Oriente. Venezuela. Disponible en: http://ri.biblioteca. udo.edu.ve/handle/123456789/2395.

7. Álvarez L. Presencia de mercurio total en habitantes de los asentamientos indígenas El Casabe, municipio de autónomo de Raúl León y el plomo, municipio autónomo Manuel Carlos Piarestado de Bolívar. Rev. Saber, Universidad de Oriente, Venezuela 2006;18(2):161-7.

8. Santos Filho E, Souza e Silva R. Sakuma M A et al. Los niveles de mercurio en el cabello de los niños que viven en Cubatão, en el sur este de Brasil.Ed. Saúde Pública 1993;27:2.
9. Instituto Nacional de Salud (Colombia). Protocolo de vigilancia y control de intoxicaciones agudas por mercurio, 2011.

10. Rice KM, Walker Jr EM, Wu M, Gillette C, Blough ER. Environmental mercury and its toxic effects. J Prev Med Public Health 2014;47(2):74-83.

\section{CORRESPONDENCIA}

Dr. Eduardo Chimenos Küstner

Campus Universitario de Bellvitge

Facultad de Odontología, $2^{a}$ planta

Feixa Llarga, s/n. 08907 Hospitalet de Llobregat

Barcelona, España

Correo electrónico: echimenos@ub.ed 